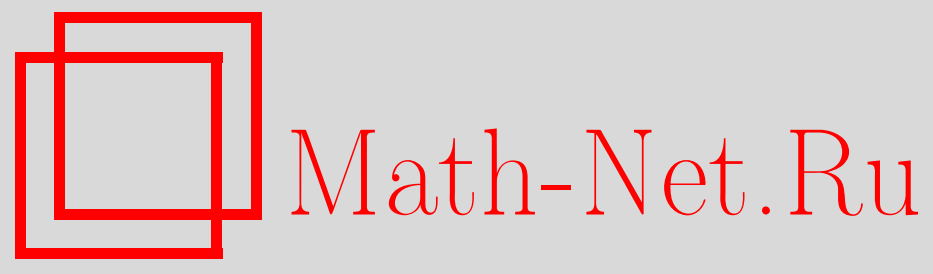

С. В. Нагаев, О вероятностных и моментных неравенствах для супермартингалов и мартингалов, Теория вероятн. и ее примен., 2006, том 51, выпуск 2, 391-400

DOI: https://doi.org/10.4213/tvp62

Использование Общероссийского математического портала Math-Net.Ru подразумевает, что вы прочитали и согласны с пользовательским соглашением

http: //www . mathnet.ru/rus/agreement

Параметры загрузки:

IP : 35.173 .137 .237

26 апреля 2023 г., 08:14:13 
9. Феллер В. Введение в теорию вероятностей и ее приложения. Т. 2. М.: Мир, 1984, $751 \mathrm{c}$.

10. Такач Л. Комбинаторные методы в теории случайных процессов. М.: Мир, 1971, $264 \mathrm{c}$.

11. Риордан Дж. Комбинаторные тождества. М.: Наука, 1982, 256 с.

Поступила в редакцию

02.VIII. 2004

Исправленный вариант

27.V.2005

(C) 2006 г.

HAГAEB C. B. ${ }^{*}$

\title{
О ВЕРОЯТНОСТНЫХ И МОМЕНТНЫХ НЕРАВЕНСТВАХ ДЛЯ СУПЕРМАРТИНГАЛОВ И МАРТИНГАЛОВ ${ }^{1)}$
}

\begin{abstract}
Доказывается вероятностное неравенство для $\max _{k \leqslant n} S_{k}$, где $S_{k}=$ $\sum_{j=1}^{k} X_{j}$ в предположении, что последовательность $S_{k}, k=\overline{1, n}$, образует супермартингал. Неравенство формулируется в терминах вероятностей $\mathbf{P}\left(X_{j}>y\right)$ и условных дисперсий случайных величин $X_{j}, j=\overline{1, n}$. В качестве простого следствия выводится известное моментное неравенство Буркхольдера. Даются численные оценки постоянных в неравенстве Буркхольдера.
\end{abstract}

Ключевые слова и фразы: математическое ожидание, мартингал, неравенство Буркхольдера, неравенства Бернштейна и БеннетаХёфдинга, неравенство Розенталя, неравенство Фука, сепарабельное банахово пространство, супермартингал, фильтрованное вероятностное пространство.

1. Введение. Формулировка и обсуждение результатов. Пусть последовательность случайных величин $S_{k}, k \geqslant 1$, образует супермартингал, заданный на фильтрованном вероятностном пространстве $\left(\Omega, \mathscr{F},\left(\mathscr{F}_{k}\right)_{k \geqslant 0}, \mathbf{P}\right)$ с $S_{0}=0$, $\mathscr{F}_{0}=\{\varnothing, \Omega\}$, т.e.

$$
\mathbf{E}\left\{S_{k} \mid \mathscr{F}_{k-1}\right\} \leqslant S_{k-1} .
$$

Положим $X_{k}=S_{k}-S_{k-1}, k \geqslant 0$. Определим случайные величины $\sigma_{k}^{2}$ равенствами

$$
\sigma_{k}^{2}=\mathbf{E}\left\{X_{k}^{2} \mid \mathscr{F}_{k-1}\right\}
$$

Обозначим

$$
B_{k}^{2}=\sum_{1}^{k} \sigma_{j}^{2}, \quad \bar{S}_{n}=\max _{1 \leqslant k \leqslant n} S_{k}, \quad \bar{X}_{n}=\max _{1 \leqslant k \leqslant n} X_{k}, \quad A_{t}=\sum_{1}^{n} \mathbf{E}\left|X_{j}\right|^{t} .
$$

Определим

$$
Q(x)=\mathbf{P}\left(\bar{X}_{n}>x\right)+\mathbf{P}\left(B_{n}>x\right) .
$$

Вероятностным неравенствам для мартингалов и семимартингалов посвящено довольно много работ, начиная с классических результатов Дуба (см. [1]).

В 1969 г. Стейгер [2] вывел для $\bar{S}_{n}$ неравенство типа Беннета-Хёфдинга при условии, что $\left|X_{i}\right|<L_{i}<\infty, \sigma_{i}^{2} \leqslant b_{i}^{2}<\infty$, где $L_{i}$ и $b_{i}^{2}$ - некоторые постоянные.

* Институт математики им. С.Л. Соболева СО РАН, просп. Академика Коптюга, 4, Университетский пр., 630090 Новосибирск, Россия; е-mail: nagaev@math.nsc.ru

1) Работа выполнена при финансовой поддержке Российского фонда фундаментальных исследований (код проекта 02-01-01252) и INTAS (код проекта 03-51-5018). 
Верхние оценки для $\mathbf{P}\left(\bar{S}_{n}>y\right)$, обобщающие соответствующие неравенства из [3], были получены в 1973 г. Фуком [4] в предположении, что для некоторой последовательности $\left(y_{k}\right)_{k \geqslant 1}, y_{k}>0$,

$$
\mathbf{E}\left\{X_{k}^{2}\left(y_{k}\right) \mid \mathscr{F}_{k-1}\right\}<d_{k}^{2}, \quad \mathbf{E}\left\{\left(X_{k}^{+}\right)^{t}\left(y_{k}\right) \mid \mathscr{F}_{k-1}\right\}<a_{k},
$$

где $d_{k}^{2}$ и $a_{k}$ постоянные, $t>2$,

$$
X_{k}(y)=\left\{\begin{array}{ll}
X_{k}, & X_{k} \leqslant y, \\
0, & X_{k}>y,
\end{array}, \quad X_{k}^{+}(y)=\max \left[0, X_{k}(y)\right] .\right.
$$

Эти условия могут показаться слишком ограничительными. Однако выяснилось, что они выполняются, в частности, для мартингала

$$
\mathbf{E}\left\{\left\|\sum_{1}^{n} X_{j}\right\| \mid \mathscr{F}_{k}\right\}
$$

где $X_{j}$ - независимые случайные величины со значениями в сепарабельном банаховом пространстве, $\mathscr{F}_{k}-\sigma$-алгебра, порожденная случайными величинами $X_{1}, X_{2}, \ldots, X_{k}$, если при этом $\mathbf{E}\left\|X_{j}\right\|^{t}<\infty, j \in \overline{1, n}$ (см. по этому поводу [5]-[7]).

Если мартингал $S_{n}$ не удовлетворяет условиям Фука, то при определенных ограничениях можно этого добиться с помошью подходящего преобразования $f\left(S_{n}\right)$. Применительно к критическому процессу Гальтона-Ватсона это делается в [8].

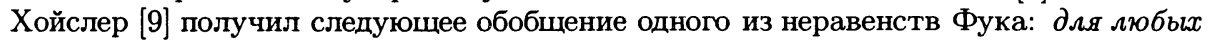
$x, u, v>0$

$$
\mathbf{P}\left(\bar{S}_{n}>x\right)<\sum_{i=1}^{n} \mathbf{P}\left(X_{i}>u\right)+\mathbf{P}\left(B_{n}>v\right)+P_{0}(x, u, v),
$$

2de

$$
P_{0}(x, u, v)=\exp \left(\frac{x}{u}\left(1-\ln \left(\frac{x u}{v^{2}}\right)\right)\right)
$$

В [10] этот результат переносится на мартингалы с непрерывным временем. В статье [11] $P_{0}$ заменяется на

$$
P_{1}(x, u, v)=\exp \left(\frac{x}{u}-\left(\frac{v^{2}}{u^{2}}+\frac{x}{u}\right) \ln \left(\frac{x u}{v^{2}}+1\right)\right) .
$$

Неравенство (2) используется в [9] для оценки скорости сходимости в функциональной предельной теореме для мартингалов с дискретным временем. Аналогичным образом используются вероятностные неравенства, полученные в [10] и [11].

В статье Пинелиса [12] даются обобщения неравенств Бернштейна и БеннетаХёфдинга на мартингалы со значениями в банаховом пространстве. Применительно к обычным мартингалам условия Пинелиса содержат ограничение $B_{n}^{2}<c<\infty$. В работе [13] одно из неравенств Фука, содержащее нормальную компоненту, переносится на банахово пространство в предположении, что

$$
\mathbf{E}\left\|X_{j}\right\|^{3}<\infty, \quad j \in \overline{1, n} .
$$

При этом на условные вторые моменты накладывается ограничение того же типа, что и у Фука. Обобшения неравенств Бернштейна и Беннета-Хёфдинга получены также в [14]-[18]. Вероятности больших уклонений $S_{n}$ изучались в [19] при условии $\max _{1 \leqslant k \leqslant n} \mathbf{E}\left|X_{k}\right|^{t}<\infty$.

В настоящей работе получен следующий результат.

Теорема 1. Пусть $0<\gamma \leqslant 1$ u $t$ удовлетворяет условию $t \geqslant \max \left(e^{6}, e^{4} / \gamma^{2}\right)$. Тогда для всех $x>0$

$$
\mathbf{P}\left(\bar{S}_{n}>x\right)<c(t, \gamma) x^{-t} \int_{0}^{x} Q\left(\varepsilon_{t} u\right) u^{t-1} d u
$$

где $\varepsilon_{t}=\ln t-2 \ln \ln t /(2 t), c(t, \gamma)=2 e^{6 \gamma t} / \gamma$.

Eсли $\varepsilon_{t}=\eta / t, \eta>0$, то неравенство (4) справедливо для всех $t>0$ с заменой $c(t, \gamma)$ на $c_{1}(t, \eta)=t e^{3 \eta \alpha(\eta)} / \eta \alpha(\eta)$, где $\alpha(\eta)=e^{\eta+1}$. 
Заметим, что

$$
\frac{1}{x^{t}} \int_{0}^{x} Q\left(\varepsilon_{t} u\right) u^{t-1} d u=\int_{0}^{1} Q\left(\varepsilon_{t} s x\right) s^{t-1} d s
$$

а значит правая часть (4) убывает по $x$.

Оценка (4) обобщает неравенство в теореме 4 статьи [3] (см. также [21, теорема 1.10]). По форме неравенство (4) близко к основному неравенству работы [23]. Метод доказательства аналогичен тому, что использовался в работах [20]-[24].

Естественно возникает вопрос, как теорема 1 соотносится с неравенством Хойслера (2). Поскольку неравенства (2) и (4) сильно отличаются по форме, сравнивать их непросто. В работе [25] показано, что (4) не является следствием (2). Аналогичные рассуждения показывают, что нельзя посредством неравенства Хойслера вывести неравенство Буркхольдера (8), которое является обобщением неравенства Розенталя [26].

Доказательство теоремы 1, содержащееся в первоначальном варианте статьи, опубликовано в [25]. В предлагаемом варианте оно усовершенствовано, а именно, из прежнего текста выделены два утверждения, которые делают изложение более прозрачным. Впрочем, последние имеют и самостоятельный интерес.

Предложение 1. При всех $x>0, y>0, \alpha>1$ выполняется неравенство

$$
f(x+\alpha y)<f(x) e^{\alpha(1-\ln \alpha)}+Q(y),
$$

где $f(x)=\mathbf{P}\left(\bar{S}_{n} \geqslant x\right)$.

Предложение 2. Если Функиия $f(x)$ не возрастает и при всех $x>0, y>0$, $\alpha>1$ удовлетворяет неравенству (5), то для любьх $\alpha>1$ и $\varepsilon>0$

$$
f(x)<\frac{\omega(\alpha, \varepsilon)}{x^{s(\alpha, \varepsilon)}} \int_{0}^{x} Q(\varepsilon u) u^{s(\alpha, \varepsilon)-1} d u,
$$

где $s(\alpha, \varepsilon)=\alpha(\ln \alpha-1) /(\varepsilon(1+\alpha \varepsilon)), \omega(\alpha, \varepsilon)=(\alpha \varepsilon)^{-1} e^{3 \alpha(\ln \alpha-1)}$.

Из теоремы 1 без труда выводятся моментные неравенства, аналогично тому, как это делается для независимых случайных величин (см. по этому поводу [20]-[23]).

Действительно, умножая обе части неравенства (4) для $t+1$ на $t x^{t-1}$ и интегрируя по $x$ от 0 до $\infty$, мы получаем следуюшее утверждение.

Следствие 1. Для любьи $t$ u $\gamma$ maжux, ито $t>\max \left(e^{6}, e^{2} / \gamma^{2}\right)-1,0<\gamma \leqslant 1$,

$$
\mathbf{E}\left\{\bar{S}_{n}^{t} ; \bar{S}_{n} \geqslant 0\right\}<c(t+1, \gamma) \varepsilon_{t+1}^{-t}\left(\bar{D}_{t}+\mathbf{E} B_{n}^{t}\right),
$$

где $\bar{D}_{t}=\mathbf{E}\left\{\bar{X}_{n}^{t} ; \bar{X}_{n} \geqslant 0\right\}$. Если $\varepsilon_{t}=\eta / t$, то неравенство (6) верно для всех $t>0 \mathrm{c}$ заменой $c(t+1, \gamma)$ на $c_{1}(t+1, \eta)$.

Если $\left(S_{k}\right)_{k \geqslant 1}$ - мартингал, то неравенства следствия 1 остаются справедливыми для $\mathrm{E}\left\{\left|\widetilde{S}_{n}\right|^{t} ; \widetilde{S}_{n} \leqslant 0\right\}$, где $\widetilde{S}_{n}=\min _{1 \leqslant k \leqslant n} S_{k}$, с заменой $\bar{D}_{t}$ на $\widetilde{D}_{t}=$ $\mathbf{E}\left\{\left|X_{n}\right|^{t} ; \min _{1 \leqslant k \leqslant n} X_{k}<0\right\}$. Суммируя неравенства для $\mathbf{E}\left\{\bar{S}_{n}^{t} ; \bar{S}_{n} \geqslant 0\right\}$ и $\mathbf{E}\left\{\left|\widetilde{S}_{n}\right|^{t} ; \widetilde{S}_{n}<0\right\}$, мы получаем следующее утверждение.

Следствие 2. Пусть $S_{n}$ - мартингал. Тогда для любьх $t>0 u \eta>0$

$$
\mathbf{E} \widehat{S}_{n}^{t}<c_{t}(\eta)\left(D_{t}+2 \mathbf{E} B_{n}^{t}\right)
$$

2дe $\widehat{S}_{n}=\max _{1 \leqslant k \leqslant n}\left|S_{k}\right|, D_{t}=\mathbf{E}\left(\max _{1 \leqslant k \leqslant n}\left|X_{k}\right|^{t}\right), c_{t}(\eta)=c_{1}(t+1, \eta)((t+1) / \eta)^{t}$.

Eсли $t>\max \left(e^{6}, e^{4} / \gamma^{2}\right)-1,0<\gamma \leqslant 1$, то неравенство (7) верно с постовнной $c_{t}^{\prime}(\gamma)=c(t+1, \gamma) \varepsilon_{t+1}^{-t}$.

Возводя обе части неравенства (7) в степень $1 / t$, имеем для $t \geqslant 1$

$$
\mathbf{E}^{1 / t} \widehat{S}_{n}^{t}<\widehat{c}_{t}\left(D_{t}^{1 / t}+2^{1 / t} \mathbf{E}^{1 / t} B_{n}^{t}\right)
$$

где $\widehat{c}_{t}=c_{t}^{1 / t}(\eta)$. Если $t>e^{6} \vee e^{4} / \gamma^{2}$, то в качестве $\widehat{c}_{t}$ можно взять $\left(c_{t}^{\prime}(\gamma)\right)^{1 / t}$. Последнее неравенство было получено Буркхольдером [27] без явного выражения для постоянной $\widehat{c}_{t}$. 
Хитченко [28] показал, что

$$
\mathbf{E} \widehat{S}_{n}^{t}<K \frac{t}{\ln t}\left(D_{t}^{1 / t}+\mathbf{E}^{1 / t} B_{n}^{t}\right)
$$

где $K-$ абсолютная постоянная (см. также [29]).

В связи с неравенством (9) представляет интерес оценка величины

$$
K_{t}=\frac{\mathbf{E}^{1 / t}\left\{\bar{S}_{n}^{t} ; S_{n} \geqslant 0\right\}}{\bar{D}_{t}^{1 / t}+\mathbf{E}^{1 / t} B_{n}^{t}} \frac{\ln t}{t} .
$$

Полагая в (8) $\widehat{c}_{t}=\left(c_{t}^{\prime}(\gamma)\right)^{1 / t}$, мы приходим к оценке

$$
\limsup _{t \rightarrow \infty} K_{t} \leqslant 2 e^{6 \gamma} .
$$

Поскольку $\gamma$ может быть сделано сколь угодно малым, мы вправе утверждать, что

$$
\limsup _{t \rightarrow \infty} K_{t} \leqslant 2
$$

В [30] показано, что $\lim _{t \rightarrow \infty} \widehat{c}_{t}((\ln t) / t)=1 / e$, если $X_{k}$ - независимые симметрично распределенные случайные величины.

3 а м е ч а н и е 1 . Поскольку

$$
\mathbf{P}\left(\bar{X}_{n}>x\right) \leqslant \sum_{1}^{n} \mathbf{P}\left(X_{j}>x\right),
$$

можно заменить $\bar{D}_{t}$ в неравенстве (6) на $A_{t}^{+}=\sum_{1}^{n} \mathrm{E}\left\{X_{j}^{t} ; X_{j} \geqslant 0\right\}$. Соответственно в неравенстве (7) можно заменить $D_{t}$ на $A_{t}$, делая его тем самым ближе по форме к неравенству Розенталя [26]. Точные оценки в неравенстве Розенталя для $\mathbf{E} S_{n}^{2 k}$, где $S_{n}$ - сумма независимых случайных величин с нулевым средним, даются в [31]. Подробный обзор моментных неравенств для мартингалов содержится в [32].

В формулируемой ниже теореме 2 даются оценки для $K_{t}$ при $t>2$.

Введем обозначения

$$
\begin{aligned}
g_{1}(t)= & \frac{2,76}{t}+\left(1+\frac{1}{t}\right) \ln (t+1)-\ln t+\ln \ln t+1,39, \\
g_{2}(t)= & \frac{3,694}{t}+\left(1+\frac{1}{t}\right) \ln (t+1)-\ln t+\ln \ln t+1,064, \\
g_{3}(t)= & \frac{1,74}{\ln t-1,1}-\left(1+\frac{1}{t}\right) \ln (\ln t-2,86)+\left(1+\frac{1}{t}\right) \ln (t+1) \\
& -\ln t+\ln \ln t+0,57\left(1+\frac{1}{t}\right)+\frac{0,57}{t}(2,86-\ln t)
\end{aligned}
$$

при $\ln t>2,86$ и $g_{3}(t)=\infty$ при $\ln t \leqslant 2,86$.

Теорема 2. Для любого $t>2$

$$
K_{t}<g(t):=\min _{1 \leqslant j \leqslant 3} e^{g_{j}(t)}
$$

Очевидно, $g_{2}(t)-g_{1}(t)=0,934 / t-0,326$. Отсюда $g_{1}(t)<g_{2}(t)$ при $t<t_{0}=\frac{0,934}{0,326} \approx$ 2,865 и $g_{1}(t)>g_{2}(t)$ при $t>t_{0}$. Заметим, что

$$
g_{1}^{\prime}(t)=\frac{1}{t \ln t}-\frac{\ln (t+1)+2,76}{t^{2}}=t^{-2}\left(\frac{t}{\ln t}-\ln (t+1)-2,76\right) .
$$

Функция $t / \ln t$ убывает при $2<t<e$. Поэтому при $2<t<e$

$$
g_{1}^{\prime}(t)<g_{1}^{\prime}(2)=4^{-1}\left(\frac{2}{\ln 2}-\ln 2-2,76\right)<0
$$


При $e<t<3$ функция $t / \ln t$ возрастает. Следовательно, для этих значений $t$

$$
g_{1}^{\prime}(t)<4^{-1}\left(\frac{3}{\ln 3}-\ln 2-2,76\right)<0 .
$$

Таким образом, мы показали, что функция $g_{1}(t)$ убывает на интервале $(2,3)$. Отсюда

$$
g_{1}(t) \wedge g_{2}(t)<g_{1}(2)=g_{1}(2) \wedge g_{2}(2) .
$$

Функция $g_{2}(t)$ убывает при $t<t_{1} \approx 20,1$. При $t>t_{1}$ она возрастает, но очень медленно. Например, $g_{2}\left(10^{8}\right) \approx 3,977$. Функция $g_{3}(t)$ убывает в области $t>e^{2,86} \approx$ 17,46 , причем $g_{3}(t)>g_{2}(t)$ для $t<t_{2} \approx 49,94$ и $g_{3}(t)<g_{2}(t)$ для $t>t_{2}$.

Таким образом,

$$
K_{t}< \begin{cases}e^{g_{1}(t)}, & 2<t<3 \\ e^{g_{2}(t)}, & 3 \leqslant t<t_{2} \\ e^{g_{3}(t)}, & t>t_{2}\end{cases}
$$

Заметим, что $g_{3}\left(t_{2}\right)<g_{2}(50) \approx 2,6<g_{2}(4) \approx 2,94$.

Из сказанного, в частности, вытекает

Следствие 3. Имеют место оченки

$$
\sup _{2<t<3} K_{t} \leqslant e^{g_{1}(2)} \approx 29, \quad \sup _{3 \leqslant t<4} K_{t} \leqslant e^{g_{2}(3)} \approx 23,1, \quad \sup _{t \geqslant 4} K_{t} \leqslant e^{g_{2}(4)} \approx 18,9 .
$$

Нетрудно видеть, что $g_{3}(t) \rightarrow 0,57$ при $t \rightarrow \infty$. Следовательно, $\lim \sup _{t \rightarrow \infty} K_{t} \leqslant$ $e^{0,57}<1,77$.

Близкие оценки в случае, когда $X_{i}$ независимы, получены в [33], а также в [34].

Теорема 1 позволяет оценивать $\mathbf{E}\left\{G\left(\bar{S}_{n}\right) ; \bar{S}_{n} \geqslant 0\right\}$ для более широкого класса функций, нежели степенные. Мы приведем один из возможных результатов такого рода.

Следствие 4. Пусть дифферениируемая фунжиия $G(x)$ с $G(0)=0$ удовлетворяет условию: существуют $t>0$ и $\alpha>0$ такие, что для любых $x>y>0$

$$
\frac{G^{\prime}(x)}{x^{t-2}}<\alpha \frac{G^{\prime}(y)}{y^{t-2}}
$$

Тогда для любого $\eta>0$

$$
\mathbf{E}\left\{G\left(\bar{S}_{n}\right) ; \bar{S}_{n} \geqslant 0\right\}<\alpha c_{1}(t, \eta)\left(\mathbf{E}\left\{G\left(\epsilon_{t}^{-1} \bar{X}_{n}\right) ; \bar{X}_{n} \geqslant 0\right\}+\mathbf{E} G\left(\epsilon_{t}^{-1} B_{n}\right)\right),
$$

где $\epsilon_{t}=\eta / t$.

В самом деле, в силу второго утверждения теоремы 1

$$
\mathbf{E}\left\{G\left(\bar{S}_{n}\right) ; \bar{S}_{n} \geqslant 0\right\}<c_{2}(t, \eta) \int_{0}^{\infty}\left(x^{-t} \int_{0}^{x} Q\left(\epsilon_{t} u\right) u^{t-1} d u\right) G_{1}^{\prime}(u) d x \equiv c_{2}(t, \eta) I_{t} .
$$

Меняя порядок интегрирования, находим

$$
\begin{aligned}
I_{t} & =\int_{0}^{\infty} u^{t-1} Q\left(\epsilon_{t} u\right)\left(\int_{u}^{\infty} x^{-t} G^{\prime}(x) d x\right) d u<\alpha \int_{0}^{\infty} Q\left(\epsilon_{t} u\right) G^{\prime}(u) d u \\
& =-\alpha \int_{0}^{\infty} G(u) d Q\left(\epsilon_{t} u\right)=\alpha\left(\mathbf{E}\left\{G\left(\epsilon_{t}^{-1} \bar{X}_{n}\right) ; \bar{X}_{n} \geqslant 0\right\}+\mathbf{E} G\left(\epsilon_{t}^{-1} B_{n}\right)\right) .
\end{aligned}
$$

Из двух последних соотношений следует нужный нам результат. цию $G$.

Неравенство типа (13) выведено в [27] при менее жестких ограничениях на функ- 
2. Доказательство предложения 1. Для любых положительных $t, s, y, C$ имеет место неравенство (см. [25, лемма 4])

$$
\mathbf{P}\left(\bar{S}_{n}>t+s+y\right)<\mathbf{P}\left(\bar{S}_{n}>t\right) P_{1}(s, y, C)+\mathbf{P}\left(B_{n}>C\right)+\mathbf{P}\left(\bar{X}_{n}>y\right),
$$

где $P_{1}(s, y, C)$ определено равенством (3). Полагая в этом неравенстве $s=(\alpha-1) y$, $C=y$, имеем $\mathbf{P}\left(\bar{S}_{n}>t+\alpha y\right)<\mathbf{P}\left(\bar{S}_{n}>t\right) P_{1}((\alpha-1) y, y, y)+Q(y)$. Нетрудно видеть, что

$$
P_{1}((\alpha-1) y, y, y)=e^{\alpha-1-\alpha \ln \alpha} \leqslant e^{\alpha(1-\ln \alpha)} .
$$

Чтобы закончить доказательство, остается подставить эту оценку в правую часть предыдушего неравенства.

3. Доказательство предложения 2. Пусть $\beta$ - любое положительное число. Рассмотрим последовательность

$$
y_{m}=(1+\alpha \varepsilon)^{m} \beta, \quad m \geqslant 1 .
$$

Заметим, что

$$
y_{m}-y_{m-1}=\alpha \varepsilon y_{m-1} .
$$

Полагая в (5) $x=y_{m-1}, y=\varepsilon y_{m-1}$, имеем при $m>1$

$$
f\left(y_{m}\right)<f\left(y_{m-1}\right) p+Q\left(\varepsilon y_{m-1}\right),
$$

где $p=p(\alpha):=\exp \{\alpha-1-\alpha \ln \alpha\}$.

Очевидно, при $\alpha>e$

$$
p(\alpha)<\rho=\rho(\alpha):=\exp \{\alpha(1-\ln \alpha)\}<1 .
$$

Поскольку $f(y) \leqslant 1$ из (16) и (17) следует, что для $m \geqslant 1$

$$
f\left(y_{m}\right)<\sum_{1}^{m-1} Q\left(\varepsilon y_{k}\right) \rho^{m-k-1}+\rho^{m-1} .
$$

В силу (14) $k=\ln \left(y_{k} / \beta\right) / \ln (1+\alpha \varepsilon)$. Отсюда

$$
\rho^{k}=\left(\frac{y_{k}}{\beta}\right)^{\ln \rho / \ln (1+\alpha \varepsilon)}
$$

Подставляя это выражение в (18), получаем оценку

$$
f\left(y_{m}\right)<\sum_{1}^{m-1} Q\left(\varepsilon y_{k}\right)\left(\frac{\beta}{y_{m-k-1}}\right)^{s(\alpha, \varepsilon)}+\left(\frac{\beta}{y_{m-1}}\right)^{s(\alpha, \varepsilon)}
$$

где $s(\alpha, \varepsilon)=-\ln \rho(\alpha) / \ln (1+\alpha \varepsilon)$. Далее, вследствие (14)

$$
\frac{\beta}{y_{m-k-1}}=\frac{1}{(1+\alpha \varepsilon)^{m-k-1}}=\frac{y_{k-1}}{y_{m+1}}(1+\alpha \varepsilon)^{\dot{3}} .
$$

Используя это равенство, (14), (15) и монотонность функций $Q(x)$ и $x^{t-1}$ получаем, что для любого $t>0$

$$
\begin{aligned}
Q\left(\varepsilon y_{k}\right)\left(\frac{\beta}{y_{m-k-1}}\right)^{t} & =(1+\alpha \varepsilon)^{3 t}\left(\frac{y_{k-1}}{y_{m+1}}\right)^{t} \frac{y_{k}-y_{k-1}}{\alpha \varepsilon y_{k-1}} Q\left(\varepsilon y_{k}\right) \\
& <\frac{(1+\alpha \varepsilon)^{3 t}}{\alpha \varepsilon y_{m+1}^{t}} \int_{y_{k-1}}^{y_{k}} Q(\varepsilon u) u^{t-1} d u
\end{aligned}
$$

Из (20) и (21) следует

$$
f\left(y_{m}\right)<\frac{\omega(\alpha, \varepsilon)}{y_{m+1}^{s(\alpha, \varepsilon)}} \int_{0}^{y_{m}} Q(\varepsilon u) u^{s(\alpha, \varepsilon)-1} d u+\left(\frac{\beta}{y_{m-1}}\right)^{s(\alpha, \varepsilon)}
$$


где

$$
\omega(\alpha, \varepsilon)=\frac{(1+\alpha \varepsilon)^{3 s(\alpha, \varepsilon)}}{\alpha \varepsilon}=\frac{1}{\alpha \varepsilon \rho^{3}} .
$$

Пусть $y_{m} \leqslant x<y_{m+1}$. Тогда ввиду (22)

$$
f(x)<f\left(y_{m}\right)<\frac{\omega(\alpha, \varepsilon)}{x^{s(\alpha, \varepsilon)}} \int_{0}^{x} Q(\varepsilon u) u^{s(\alpha, \varepsilon)-1} d u+\left(\frac{(1+\alpha \varepsilon)^{2} \beta}{x}\right)^{s(\alpha, \varepsilon)}
$$

Отсюда в силу произвольности $\beta$

$$
f(x)<\frac{\omega(\alpha, \varepsilon)}{x^{s(\alpha, \varepsilon)}} \int_{0}^{x} Q(\varepsilon u) u^{s(\alpha, \varepsilon)-1} d u .
$$

Предложение 2 доказано.

4. Доказательство теоремы 1. Доказательство сводится к оценке величин $\omega(\alpha, \varepsilon)$ и $s(\alpha, \varepsilon)$ в неравенстве (24) при специальном выборе параметров $\alpha$ и $\varepsilon$. Положим $\alpha=\gamma t /(\ln t-2 \ln \ln t)$, где $\gamma-$ положительное число, удовлетворяюшее единственному ограничению $\gamma \leqslant 1$. При $\varepsilon=\left(\frac{1}{2}\right)(\ln t-2 \ln \ln t) / t$ имеет место неравенство (см. [25, формула (31)])

$$
\frac{1}{\rho^{3} \alpha \varepsilon}<\frac{2 e^{6 \gamma t}}{\gamma} \text {. }
$$

Кроме того,

$$
s(\alpha, \varepsilon)>1
$$

$[25$, формула(30)].

В силу предложений 1 и 2 функция $f(x)=\mathbf{P}\left(\bar{S}_{n}>x\right)$ удовлетворяет неравенству (24). Поэтому из (23)-(26) следует неравенство (4). Обратимся теперь к случаю $\varepsilon_{t}=\eta / t$. Легко видеть, что

$$
s\left(\alpha(\eta), \frac{\eta}{t}\right)=\frac{\alpha(\eta) \eta}{\ln (1+\alpha(\eta) \eta / t)}>t .
$$

С другой стороны, в рассматриваемом случае

$$
\omega\left(\alpha, \varepsilon_{t}\right)=\frac{t e^{3 \alpha(\eta) \eta}}{\alpha(\eta) \eta}
$$

Из (24), (27), (28) следует второе утверждение теоремы.

5. Доказательство теоремы 2. Согласно второму неравенству следствия 1 ,

$$
\mathbf{E}^{1 / t}\left\{\bar{S}_{n}^{t} ; \bar{S}_{n} \geqslant 0\right\}<c_{2}^{1 / t}(t, \eta) \frac{t}{\ln t}\left(\bar{D}_{t}^{1 / t}+\mathbf{E}^{1 / t} B_{n}^{t}\right)
$$

где

$$
c_{2}(t, \eta)=\frac{e^{3 \eta \alpha(\eta)}(t+1)^{t+1}(\ln t)^{t}}{\alpha(\eta) \eta^{t+1} t^{t}}
$$

Нетрудно показать, что

$$
c_{2}^{1 / t}(t, \eta)=\exp \left\{\frac{f(t, \eta)}{t}+\left(1+\frac{1}{t}\right) \ln (t+1)-\ln t+\ln \ln t\right\}
$$

где $f(t, \eta)=3 \eta \alpha(\eta)-(t+1) \ln \eta-\ln \alpha(\eta)$. Обозначим

$$
\omega_{t}=\min _{\eta} f(t, \eta)
$$

Пусть $\eta_{t}-$ значение $\eta$, при котором достигается $\omega_{t}$, т.е. $\omega_{t}=f\left(t, \eta_{t}\right)$. Нетрудно видеть, что $\eta_{t}$ удовлетворяет уравнению $f_{\eta}^{\prime}(t, \eta)=0$. Это уравнение можно записать в виде

$$
(\eta+1)\left(3 \eta e^{\eta+1}-1\right)=t
$$


Функция, стоящая в левой части уравнения (32), строго возрастает при $\eta>0$ и стремится к бесконечности при $\eta \rightarrow \infty$. Кроме того, она отрицательна в нуле. Поэтому уравнение (32) имеет единственное решение при любом $t>0$.

Приближенное значение $\eta_{2} \approx 0,249$, соответственно, $-\ln \eta_{2} \approx 1,39$. Далее, $\eta_{4} \approx 0,345,-\ln \eta_{4} \approx 1,064$. Все численные значения даются здесь с недостатком.

Заметим, что в силу (32)

$$
3 \eta e^{\eta+1}=\frac{t}{\eta+1}+1
$$

Отсюда

$$
f(t, \eta)=\frac{t}{\eta+1}-(t+1) \ln \eta-\eta .
$$

Рассмотрим функцию

$$
f_{s}(t)=\frac{\omega_{s}}{t}+\left(1+\frac{1}{t}\right) \ln (t+1)-\ln t+\ln \ln t
$$

зависящую от параметра $s \geqslant 2$. Ввиду $(31) c_{2}^{1 / t}\left(t, \eta_{s}\right)=e^{f_{s}(t)+(s / t-1) \ln \eta_{s}}$. Согласно определению $f_{s}(t)$,

$$
f_{s}(t)+\left(\frac{s}{t}-1\right) \ln \eta_{s}=\frac{\omega_{s}+(s-t) \ln \eta_{s}}{t}+\left(1+\frac{1}{t}\right) \ln (1+t)-\ln t+\ln \ln t .
$$

Подставляя в (33) найденные выше значения $\eta_{s}, \ln \eta_{s}, s=2,4$, находим, чาо

$$
\omega_{2}<5,54, \quad \omega_{4}<7,955 .
$$

Возврашаясь теперь к (34), мы заключаем, что $f_{s}(t)+(s / t-1) \ln \eta_{s}<g_{s}(t), s=2,4$. В результате мы получаем оценку

$$
K_{t} \leqslant c_{2}^{1 / t}\left(t, \eta_{2}\right) \wedge c_{2}^{1 / t}\left(t, \eta_{4}\right) \leqslant e^{g_{1}(t)} \wedge e^{g_{2}(t)} .
$$

Если $\eta$ удовлетворяет уравнению (32), то $(\eta+1)^{2} e^{\eta+1}>t / 3$. Полагая $y=\eta+1$, имеем $e^{y+2 \ln y}>3^{-1} t$, т.е. $y+2 \ln y>\ln \left(3^{-1} t\right)$. Отсюда

$$
y>\ln \left(3^{-1} t\right)\left(1+\frac{2 \ln y}{y}\right)^{-1}>c_{0}^{-1} \ln \left(3^{-1} t\right),
$$

где $c_{0}=1+2 / e$, поскольку

$$
\max _{y>0} \frac{\ln y}{y}=\frac{1}{e}
$$

Ввиду (36) при $t>3$

$$
\frac{1}{\eta+1}<\frac{c_{0}}{\ln t-\ln 3}<\frac{1,74}{\ln t-1,1}
$$

и

$$
\eta>0,57(\ln t-\ln 3)-1>0,57(\ln t-2,86) \text {. }
$$

Наконец, в силу (38) при $\ln t>2,86$

$$
\ln \eta>\ln (\ln t-2,86)-0,57 \text {. }
$$

Из (33) и (37)-(39) следует оценка

$$
\frac{f(t, \eta)}{t}<\frac{1,74}{\ln t-1,1}-\left(1+\frac{1}{t}\right) \ln (\ln t-2,86)+\frac{0,57}{t}(2,86-\ln t)+0,57\left(1+\frac{1}{t}\right)
$$

при условии, чтоln $t>2,86$. Подставляя эту оценку в правую часть (31), имеем при $\ln t>2,86$

$$
c_{3}^{1 / t}(t, \eta)<e^{g_{3}(t)} .
$$

Комбинируя оценки (35) и (40), мы получаем желаемый результат.

В заключение автор выражает благодарность рецензенту, который обратил его внимание на работу [11], а также Э.Л. Пресману, чьи замечания позволили сушественно улучшить изложение. 


\section{СПИСОК ЛИТЕРАТУРЫ}

1. Дуб Дж. Л. Вероятностные процессы. М.: ИЛ, 1956, 605 с.

2. Steiger $W$. A best possible Kolmogoroff type inequality for martingales and characteristic property. - Ann. Math. Statist., 1969, v. 40, № 3, p. 764-769.

3. Фук Д. Х., Нагаев С. В. Вероятностные неравенства для сумм независимых случайных величин. - Теория вероятн. и ее примен., 1971, т. 16, в. 4, с. 660-675.

4. Фук Д. Х. Некоторые вероятностные неравенства для мартингалов. - Сиб. матем. журн., 1973, т. 14, с. 185-193.

5. Юринский В. В. Показательные оценки для больших уклонений. - Теория вероятн. и ее примен., 1974, т. 19 , в. 1, с. $152-154$.

6. Нагаев С. В., Пинелис И. Ф. О больших уклонениях для сумм независимых случайных величин со значениями в банаховом пространстве. - Тезисы докладов 2-й Вильнюсской конференции по теории вероятностей и математической статистике, т. 2, Вильнюс, 1977 , с. 66-67.

7. Володин Н.А., Морозова Л. Н. Некоторые оценки вероятностей больших уклонений для мартингалов и сумм случайных векторов. - Вероятностные процессы и математическая статистика. Ташкент: Фан, 1978, с. 35-43.

8. Нагаев С. В., Вахтель В.И. Вероятностные неравенства для критического процесса Гальтона-Ватсона. - Теория вероятн. и ее примен., 2005, т. 50, в. 3, с. 266291.

9. Haeusler E. An exact rate of convergence in the functional central limit theorem for special martingale difference arrays. - Z. Wahrscheinlichkeitstheor. Verw. Geb., 1984, v. 65 , № 4 , p. 523-534.

10. Kubilius K., Mémin J. Inégalité exponentielle pour les martingales locales. C. R. Acad. Sci. Paris, 1994, v. 319, № 7, p. 733-738.

11. Courbot B. Rates of convergence in the functional CLT for martingales. - C. R. Acad. Sci. Paris, 1999, v. 328, №6, p. 509-513.

12. Pinelis $I$. Optimum bounds for the distributions of martingales in Banach spaces. Ann. Probab., 1994, v. 22, № 4, p. 1679-1706.

13. Dehling H., Utev S. A. An exponential inequality for martingales. - Siberian Adv. Math., 1993, v. 3, № 3, p. 197-203.

14. Azuma $K$. Weighted sums of certain dependent random variables. - Tôhoku Math. J., 1967, v. 19 , p. 357-367.

15. Van de Geer $S$. Exponential inequalities for martingales' with applications to maximum likelihood estimation for counting process. - Ann. Statist., 1995, v. 23, № 5, p. 1779-1801.

16. De la Peña V.H. A general class of exponential inequalities for martingales and ratios. - Ann. Probab., 1999, v. 27, № 1, p. 537-564.

17. Dzhaparidze K., van Zanten $J$. H. On Bernstein-type inequalities for martingales. Stochastic Process. Appl., 2001, v. 93, № 1, p. 109-117.

18. Bentkus V. On Hoeffding's inequalities. - Ann. Probab., 2004, v. 32, № 2, p. 16501673.

19. Lesigne E., Volný D. Large deviations for martingales. - Stochastic Process. Appl., 2001, v. 96, № 1, p. 143-159.

20. Нагаев С. В., Пинелис И. Ф. Некоторые неравенства для распределений сумм независимых случайных величин. - Теория вероятн. и ее примен., 1977, т. 22, в. 2, c. $254-263$.

21. Nagaev S. V. Large deviations of sums of independent random variables. - Ann. Probab., 1979, v. 7, № 5, p. 745-789.

22. Нагаев С. В. Вероятностные неравенства для сумм независимых случайных величин со значениями в банаховом пространстве. - Труды Ин-та математики СО AH CCCP, 1982, т. 1, c. 159-167.

23. Нагаев С. В. Вероятностные неравенства для сумм независимых случайных величин в банаховом пространстве. - Сиб. матем. журн., 1987, т. 28, № 4, с. 171-184.

24. Нагаев С. В. О вероятностных и моментных неравенствах для сумм зависимых случайных величин. - Теория вероятн. и ее примен., 2000, т. 45, в. 1, с. 194-202. 
25. Nagaev S. V. On probability and moment inequalities for supermartingales and martingales. - Acta Appl. Math., 2003, v. 79, № 1-2, p. 35-46.

26. Rosenthal H. P. On the subspaces of $L^{p}(p>2)$ spanned by sequences of independent random variables. - Israel J. Math., 1970, v. 8, p. 273-303.

27. Burkholder D. L. Distribution function inequalities for martingales. - Ann. Probab., 1973 , v. 1, p. $19-42$.

28. Hitczenko $P$. Best constants in martingale version of Rosenthal's inequality. - Ann. Probab., 1990, v. 18, № 4, p. 1656-1668.

29. Hitczenko $P$. Upper bounds for the $L_{p}$-norms of martingales. - Probab. Theory Related Fields, 1990 , v. 86 , № 2, p. 225-238.

30. Ибрагимов P., Шарахметов III. О точной константе в неравенстве Розенталя. Теория вероятн. и ее примен., 1997, т. 42 , в. 2 , с. $341-350$.

31. Ибрагимов P., Шарахметов Ш. Точная константа в неравенстве Розенталя для случайных величин с нулевым средним. - Теория вероятн. и ее примен., 2001, т. 46 , в. 1 , c. $134-138$.

32. Пешкир $Г$., Ширяев $A . H$. Неравенства Хинчина и мартингальное расширение сферы их действия. - Успехи матем. наук, 1995, т. 50, № 5, с. 3-62.

33. Johnson W. B., Schechtman G., Zinn J. Best constants in moment inequalities for linear combinations of independent and exchangeable random variables. - Ann. Probab., 1985 , v. 13, № 1 , p. 234-253.

34. Нагаев С. В. Некоторые уточнения вероятностных и моментных неравенств. Теория вероятн. и ее примен., 1997, т. 42, в. 4, с. 832-838.

Поступила в редакцию

11.VI.2002

Исправленный вариант

14.IV.2005

(C) $2006 \mathrm{r}$.

ЯРЫКИН П. Н.*

\title{
УСТОЙЧИВОСТЬ НЕЛИНЕЙНОГО СТОХАСТИЧЕСКОГО ПРОЦЕССА, АППРОКСИМИРУЮЩЕГО СИСТЕМУ ВЗАИМОДЕЙСТВУЮЩИХ ЧАСТИЦ
}

\begin{abstract}
Рассматривается нелинейное стохастическое дифференциальное уравнение типа Маккина-Власова без «внешнего поля». Доказывается существование и сильная единственность решения уравнения. Далее доказывается существование и слабая единственность стационарного решения в классе распределений с заданным математическим ожиданием, а также сходимость по вероятности любого решения к стационарному распределению с соответствующим математическим ожиданием при больших временах.
\end{abstract}

Ключевые слова и фразы: нелинейный стохастический процесс, уравнение Маккина-Власова, устойчивость.

Введение Настоящая статья посвящена исследованию решения нелинейного стохастического дифференциального уравнения (СДУ) вида

$$
\tilde{X}_{t}=X_{0}+W_{t}+\int_{0}^{t} \int_{\mathbf{R}^{1}} b\left(\tilde{X}_{s}, y\right) \mu_{s}^{\tilde{X}}(d y) d s, \quad t \geqslant 0,
$$

где $\mu_{s}^{\tilde{X}}$ является распределением $\widetilde{X}_{s} \in \mathbf{R}^{1}, W_{t}$ - броуновское движение, не зависящее от начального значения $X_{0}, b\left(\tilde{X}_{s}, y\right)$ - функция попарного взаимодействия.

* Московский государственный университет им. М.В. Ломоносова, механикоматематический факультет, кафедра теории вероятностей, Ленинские горы, 119992 Москва, Россия; e-mail: yarykin@plushka.msk.ru 